\title{
A protocol for investigation of the effects of outdoor air pollution on stroke incidence, phenotypes and survival using the South London Stroke Register
}

\author{
Ravi Maheswaran*1, Tim Pearson1, Michael J Campbell2, Robert P Haining3, \\ Cameron W McLeod ${ }^{4}$, Nigel Smeeton ${ }^{5}$ and Charles DA Wolfe ${ }^{5}$
}

Address: ${ }^{1}$ Public Health GIS Unit, School of Health and Related Research, University of Sheffield, Regent Court, 30 Regent Street, Sheffield S1 4DA, UK, ${ }^{2}$ Health Services Research Unit, School of Health and Related Research, University of Sheffield, Regent Court, 30 Regent Street, Sheffield S1 4DA, UK, ${ }^{3}$ Department of Geography, University of Cambridge, Downing Place, Cambridge CB2 3EN, UK, ${ }^{4}$ Centre for Analytical Sciences, Department of Chemistry, University of Sheffield, Western Bank, Sheffield S10 2TN, UK and ${ }^{5}$ Department of Public Health Sciences, Division of Health and Social Care Research, King's College, London, 5th Floor Capital House, 42 Weston Street, London SE1 3QD, UK

Email: Ravi Maheswaran* - r.maheswaran@sheffield.ac.uk; Tim Pearson - t.pearson@sheffield.ac.uk;

Michael J Campbell - m.j.campbell@sheffield.ac.uk; Robert P Haining - rph26@cam.ac.uk; Cameron W McLeod - C.W.McLeod@sheffield.ac.uk; Nigel Smeeton - nigel.smeeton@kcl.ac.uk; Charles DA Wolfe - charles.wolfe@kcl.ac.uk

* Corresponding author

Published: 17 March 2006

International Journal of Health Geographics2006, 5:10 doi:10.1 186/1476-072X-5-10

This article is available from: http://www.ij-healthgeographics.com/content/5/I/10

(c) 2006Maheswaran et al; licensee BioMed Central Ltd.

This is an Open Access article distributed under the terms of the Creative Commons Attribution License (http://creativecommons.org/licenses/by/2.0), which permits unrestricted use, distribution, and reproduction in any medium, provided the original work is properly cited.
Received: 05 December 2005

Accepted: 17 March 2006

\begin{abstract}
Stroke is a major cause of death and disability. About 5.3 million people die every year from stroke worldwide with over 9 million people surviving at any one time after suffering a stroke. About I in 4 men and I in 5 women aged 45 years will suffer a stroke if they live to their 85th year. It is estimated that by 2023 there will be an absolute increase in the number of people experiencing a first ever stroke of about 30\% compared with 1983.

In the UK, stroke is the third commonest cause of death and the most common cause of adult physical disability and consumes $5 \%$ of the health and social services budget. Stroke is assuming strategic public health importance because of increased awareness in society, an ageing population and emerging new treatments. It is an NHS health service and research priority, being identified as a target in Our Healthier Nation and the NSF for Older People for prevention and risk factor control and in the NHS Plan as a disease requiring intermediate care planning and reduction in inequalities of care.
\end{abstract}

Whilst a number of risk factors for stroke are well known (e.g. increasing age, ethnicity, socioeconomic deprivation, hypertension), the potential importance of outdoor air pollution as a modifiable risk factor is much less well recognised. This is because studies to date are inconclusive or have methodological limitations. In Sheffield, we estimated that II\% of stroke deaths may be linked to current levels of outdoor air pollution and this high figure is explained by the fact that so many people are exposed to air pollution.

We plan to study the effects of outdoor air pollution on stroke using a series of epidemiological (i.e. population based) studies. The purpose of this project is:

- to examine if short term increases in pollution can trigger a stroke in susceptible individuals; 


\begin{abstract}
- to investigate if the occurrence of stroke is higher amongst people living in more polluted areas (which would be explained by a combination of exposure to short term increases and longer term exposure to higher pollution levels); and
\end{abstract}

- to see if people living in more polluted areas have reduced survival following their stroke.

We will use geographical information systems, robust statistical methods and powerful grid computing facilities to link and analyse the data. The datasets we will use are the South London Stroke Register database, daily monitored pollution data from national monitoring networks and modelled pollution data for London from the Greater London Authority. The South London Stroke Register records information on all patients who suffer a stroke ("incident" cases) living within a defined area. This stroke incidence dataset offers major advantages over previous studies examining the effects of pollution on hospital admissions and mortality, as not all patients with stroke are admitted or die and there may be a delay between the onset of stroke and admission or death. In addition, it contains other useful information, particularly the type of stroke people have suffered.

Air pollution is a potentially modifiable risk factor for stroke. This study will provide robust population level evidence regarding the effects of outdoor air pollution on stroke. If it confirms the link, it will suggest to policy-makers at national and international levels that targeting policy interventions at high pollution areas may be a feasible option for stroke prevention.

\section{Purpose of proposed investigation}

There is increasing interest in the association between outdoor pollution and stroke and growing recognition that it may be a more important factor in terms of population attributable risk than previously recognised. To date, however, studies have been relatively small scale in size with inconclusive results and have not examined in detail the differential effects on stroke phenotypes. The purpose of this project is to examine the acute effects of outdoor air pollution on incident stroke and stroke phenotypes, investigate stroke incidence rates in high pollution areas which would be due to a combination of acute and chronic exposure effects, and examine if high outdoor air pollution levels reduce survival following stroke.

We plan to use case-crossover methodology, Bayesian hierarchical methods for spatial studies and survival analysis to examine these issues. The datasets we will use are the South London Stroke Register data which records incident stroke cases, daily monitored pollution data from national monitoring networks and modelled pollution data for London.

It is estimated that there are 5.3 million deaths a year from stroke worldwide and over 9 million survivors at any one time [1]. Almost one in four men and one in five women aged 45 will have a stroke if they live to their 85 th year. The overall incidence is around 2 to 5 per thousand population. It is estimated that by 2023 there will be an absolute increase in the number of patients experiencing a first ever stroke of about $30 \%$ compared with 1983 . There is a total prevalence rate of around 5 per thousand [1].
Stroke is the third most common cause of death and most common cause of adult physical disability in the UK and consumes $5 \%$ of the health and social services budget. Stroke is assuming strategic public health importance because of increased awareness in society, an ageing population and emerging new treatments. It is an NHS health service and research priority, being identified as a target in Our Healthier Nation and the NSF for Older People for prevention and risk factor control and in the NHS Plan as a disease requiring intermediate care planning and reduction in inequalities of care.

Whilst a number of risk factors for stroke are well known (e.g. age, ethnicity, deprivation, hypertension), the potential importance of outdoor air pollution as a modifiable risk factor is much less well recognised. We calculated that in Sheffield, 11\% of stroke deaths may be attributable to outdoor air pollution and this high figure is explained by the fact that outdoor air pollution is a prevalent exposure [2]. Many of the numerous studies on the acute health effects of outdoor air pollution have not specifically examined stroke. In contrast, two high profile case-crossover studies $[3,4]$ examining 772 and 691 myocardial infarction cases respectively, have contributed to establishing outdoor air pollution as a recognised trigger for myocardial infarction.

\section{Background to the programme of work Acute effects of air pollution on stroke}

Early studies examining severe pollution episodes reported increased mortality from stroke [5,6], although one report found no increase in requests for hospital admission for cerebral haemorrhage [7]. Whilst pollution 
levels have reduced substantially, numerous daily time series studies have found associations between current levels of outdoor air pollutants and total and cardiorespiratory mortality. A number of studies on daily air pollution have specifically reported on stroke mortality, with several finding an association [8-13] but not others $[14,15]$. Similarly, several studies examining daily hospital admissions for stroke have observed adverse effects [16-20] while others have not $[21,22]$. However, reporting bias, i.e. an increased likelihood of reporting positive studies, cannot be ruled out. Several studies used time series analyses, while one used a case-crossover study design [20]. The pollutants for which studies have found associations include sulphur dioxide $\left(\mathrm{SO}_{2}\right)$, nitrogen oxides $\left(\mathrm{NO}_{\mathrm{x}}\right)$, carbon monoxide $(\mathrm{CO})$, ozone $\left(\mathrm{O}_{3}\right)$ and particulate matter $\left(\mathrm{PM}_{10}\right)$. However, studies reporting no evidence of association have also examined these pollutants. In two pollutant models examining stroke admissions using a case-crossover approach, Tsai [20] reported that the effects were mainly seen for $\mathrm{PM}_{10}$ and $\mathrm{NO}_{2}$, while Kan [11] encountered collinearity problems in multiple pollutant models in a time series study.

One study specifically examined sex differences in the air pollution effect and found that its effect on stroke mortality was only seen in women [10]. With regard to age, a study reported that the association with stroke mortality was only seen in people aged over 65 years [10] while another found associations with stroke admissions in people aged 20-64 and 65+ years [18]. The effect of $\mathrm{NO}_{2}$ and $\mathrm{PM}_{10}$ on stroke admissions was only apparent in warm weather $\left(>20^{\circ} \mathrm{C}\right)$ in one study [20] while in another, an $\mathrm{NO}_{2}$ effect was observed in warm and cool weather while a $\mathrm{CO}$ effect was only seen in warm weather [19]. There is a strong association between socioeconomic deprivation and stroke [23] but effect modification by deprivation on the association between air pollution and stroke has not been examined. With respect to potential mechanisms, one hypothesis is that air pollution causes increased coagulability [24], which could explain ischaemic stroke. One study reported a significant association with only ischaemic stroke mortality [12] whilst another reported associations with both haemorrhagic and ischaemic stroke admissions [20]. However, no study has examined the association with clinical or aetiological phenotypes. We propose to examine the above issues using the South London Stroke Register dataset. This stroke incidence dataset offers major advantages over studies examining acute effects of pollution on hospital admissions and mortality, as not all patients with stroke are admitted or die and there may be a delay between stroke onset and admission or death.

\section{Stroke incidence rates in high pollution areas}

Few studies have examined stroke mortality and hospital admission rates, and none have studied incidence rates, in areas with high levels of outdoor air pollution. In a national study, we found that stroke mortality risk was $5 \%$ higher in areas within $200 \mathrm{~m}$ of a main road, the latter used as a proxy for road traffic pollution [25]. We estimated that 990 stroke deaths per year in England and Wales would have been attributable to road traffic pollution if a causal link were assumed. In a small area level ecological correlation study we recently completed in Sheffield, we found that stroke mortality in the most polluted fifth of the city was 37\% higher than that in the least polluted fifth of the city [2]. Overall, $11 \%$ of stroke deaths would have been attributable to outdoor air pollution. Elevated stroke mortality rates associated with outdoor air pollution in these studies would be due to a combination of its acute and chronic exposure effects.

We also found an association with stroke emergency hospital admission rates but the association was not as strong (13\% increase in rate ratio for $\left.\mathrm{NO}_{\mathrm{x}}\right)$ [2]. This may be because the spatial variation, even for emergency admissions, is influenced by a variety of factors including local medical practice and bed availability, and these could have masked the magnitude of the underlying association. Investigation of the association between outdoor air pollution and stroke incidence rates, as we propose to do in this project, should not be affected by factors influencing hospital admission. In addition, we will be able to examine associations with stroke phenotypes as these data are available in the South London Stroke Register dataset.

\section{Outdoor air pollution and survival following stroke}

The association between daily variation in outdoor air pollution levels and stroke is generally clearer for stroke mortality than for hospital admissions. One of the possible explanations is that increases in outdoor air pollution may precipitate death in patients who have already sustained a stroke with resulting compromised respiratory function. Schwartz reported that amongst people dying of stroke on high pollution days, there was a higher level of respiratory disease recorded as a contributory factor compared with people dying of stroke on low air pollution days [26]. Decreased ventilatory function has also been found to be a risk factor for fatal stroke though the mechanism is not clear [27]. Linkage of average outdoor air pollution levels to the South London Stroke dataset will allow examination of the hypothesis that people with stroke living in polluted areas have reduced survival after adjusting for a range of individual level prognostic factors. 


\section{Aim and objectives}

The aim of this project is to examine the association between outdoor air pollution levels and stroke using a series of epidemiological studies. The objectives are:

1. To examine acute effects of particulate $\left(\mathrm{PM}_{10}\right.$, black smoke) and gaseous $\left(\mathrm{NO}_{x^{\prime}} \mathrm{SO}_{2}, \mathrm{CO}, \mathrm{O}_{3}\right)$ outdoor air pollutants on stroke incidence;

2. To examine if the above effects are modified by age, sex, temperature or socioeconomic deprivation;

3. To examine the differential effects of particulate and gaseous pollutants on incidence using multi-pollutant models and control by matching;

4. To examine the acute effects of outdoor air pollutants on incident stroke phenotypes (pathological, clinical and aetiological subtypes);

5. To examine if stroke incidence rates, and incidence rates by phenotype, are higher in more polluted areas due to the combined effects of acute and chronic exposure;

6. To examine if outdoor air pollution levels influence survival following stroke;

7. To apply Bayesian methodology to the above models to incorporate uncertainty in exposure measurement and obtain more robust effect estimates.

\section{Design and methods Study designs \\ Case-crossover study}

Maclure developed the case-crossover design in 1991 as a method for investigating the acute effects of an exposure [28]. This study design offers an elegant alternative to statistical time series modelling for examining short-term environmental effects on health. The case-crossover approach is similar in some conceptual aspects to a matched case-control study. Each individual who has had an event at a particular time is matched to one or more time periods close to the event time when he or she did not have the event (i.e. control periods). The individual's exposures at the time of the event are compared with those of control periods. Each risk set thus comprises a single individual who crosses over between different exposure levels in the case and control time periods. These matched pairs are analysed using conditional logistic regression. A major advantage is that each subject serves as his or her own control and the use of one or more control periods close to the event period means that all covariates that could change slowly over time, e.g. age, socioeconomic status, smoking, are controlled for by matching. Another advantage is that the case-crossover approach allows for control of seasonal variation and time trends in the design phase. It has been demonstrated that even very strong confounding of exposure by seasonal patterns can be controlled for by this method providing control days are adequately sampled to avoid subtle biases [29].

\section{Small area level ecological correlation study}

We will use this study design to examine if stroke incidence rates, and the incidence rates by phenotype, are higher in more polluted areas due to the combined effects of acute and chronic exposure. 2001 census output areas will be used as the units of analysis. Whilst ecological studies have recognised limitations, the use of small geographical areas as the units for analysis will address some of these limitations. Small area level studies are able to capture fine grain variation in outdoor air pollution levels. In addition, population characteristics, including exposure levels and socioeconomic factors, are likely to be more homogenous within small geographical areas. The analysis should assess spatial autocorrelation in model residuals between neighbouring areas which could bias results and we plan to do this using conditional autoregressive spatial models.[30,31] We have used this study design in several projects examining the impact of environmental and socioeconomic factors on health and healthcare.

\section{Cohort study}

A cohort study design will be used to examine if outdoor air pollution levels influence survival following stroke. The cohort will comprise patients recorded on the South London Stroke Register. We will use modelled pollution data to attribute an average exposure level to individuals within the cohort. The register tracks residential changes of cohort members within the study area and this information may be used to take account of varying levels of pollution exposure due to changes in residence. Exposure at each location will be weighted by the time spent at that location. Follow up will be from onset of the first stroke to death, end of study period or a move out of the area. The analysis will use standard Cox's proportional hazards modelling to assess the effect of outdoor air pollution on survival.

\section{Air pollution data \\ Monitored data}

Daily outdoor levels of $\mathrm{SO}_{2}, \mathrm{NO}_{\mathrm{x}^{\prime}} \mathrm{CO}, \mathrm{O}_{3}$ and $\mathrm{PM}_{10}$ from 1996-2003 will be obtained from Department for Environment Food and Rural Affairs (DEFRA). DEFRA co-ordinates the funding of three automated monitoring networks on behalf of the Government and the devolved administrations - the Automatic Urban Network (AUN), the Automatic Rural Network (ARN) and the Hydrocarbon Network. The number of automatic network sites increased from 74 at the end of 1996 to 116 by June 2003. 
The increase was mainly in urban sites to provide more comprehensive coverage on air quality in UK cities. The AUN monitors $\mathrm{SO}_{2}, \mathrm{NO}_{\mathrm{x}}, \mathrm{CO}, \mathrm{O}_{3}$ and $\mathrm{PM}_{10}$ at a variety of urban locations, including urban background locations representative of population exposure for significant periods, and "hotspot" monitoring at urban kerbsides and in the vicinity of industrial sources. The ARN sites mainly monitor $\mathrm{O}_{3}$ but also $\mathrm{SO}_{2}, \mathrm{NO}_{\mathrm{x}}$ and $\mathrm{PM}_{10}$ in some cases. Quality control arrangements ensure that measurement precision standards of at least $\pm 10 \mu \mathrm{g} / \mathrm{m}^{3}$ for $\mathrm{SO}_{2} \pm 8 \mu \mathrm{g} /$ $\mathrm{m}^{3}$ for $\mathrm{NO}_{\mathrm{x}}$ and $\mathrm{O}_{3^{\prime}} \pm 0.5 \mathrm{mg} / \mathrm{m}^{3}$ for CO and $\pm 4 \mu \mathrm{g} / \mathrm{m}^{3}$ for $\mathrm{PM}_{10}$ are achieved at automatic monitoring sites. We will also use data from a non-automatic network, the UK Smoke and Sulphur Dioxide Network. Measurements of daily $\mathrm{SO}_{2}$ and black smoke concentrations in urban areas are currently made through this Network, which provides a long-term database for these pollutants to assess trends in concentration and spatial distribution. The use of these data would potentially increase coverage but the limitation is the range of pollutants measured.

\section{Modelled data}

Validated modelled outdoor air quality data for $\mathrm{NO}_{2}$ and $\mathrm{PM}_{10}$ will be obtained from the Greater London Authority. Data are available for 1999 and should also become available for 2001. These were produced to inform the Air Quality Strategy for Greater London which deals mainly with ambient, or outdoor, air quality. Air quality was assessed by calculating the amount of air pollution at ground level as an annual average concentration. The model took into account:

- emissions from cars and other vehicles, industrial processes, boilers (such as those used for central heating), construction and various other processes;

- the weather, local geography and topography (shape of the ground's surface, e.g. hills) and the height of the emissions;

- chemical reactions of emissions from these sources in the atmosphere which can create further pollution (i.e. secondary pollution);

- pollution travelling into London from the rest of the UK and overseas (i.e. trans-boundary pollution).

The modelled data were generated at a 10 metre grid square resolution and will be used in the ecological and cohort studies.

\section{Meteorological data}

Data on daily temperature, relative humidity, wind-speed and wind-chill from 1996-2003 will be obtained from the Meteorological Office. These data are required to adjust for potential confounding of the association between air pollution and stroke due to these climate related factors that may vary on a daily basis.

\section{South London Stroke Register data}

The South London Stroke Register is a research register of international standing covering a multi ethnic inner city population of 235,000, which has been ongoing since 1995 [32]. Its aims are to develop chronic disease methodology (case ascertainment, multi level modelling for outcome assessment) and prospectively estimate the impact of disease (incidence and outcome), analyse the predictors of risk and outcome, monitor trends in incidence and survival and estimate and model the resource use and cost of care. The population covers residents in 22 wards of South London. Data are collected prospectively by the registry team, comprising 2 research associates supported by a research stroke physician, a coordinator, trained students and study investigators. Twelve overlapping sources of notification are used to ascertain cases. Hospital surveillance of stroke admissions includes 5 hospitals and checks on health authority activity data outside these hospitals. Death certificates are checked for potential cases that are validated according to clinical registration criteria. ONS notify the register of any patient who dies. Because of the possibilities of under-ascertainment with registers, intense feedback of results and communication with the 140 general practices involved in registering patients is carried out using visits, phone, letter, emails and newsletters. This involves the research associates and a register coordinator. All patients are assessed within 48 hours of the stroke by a study physician. Data collected at the initial assessment are:

Demography - age, sex, ethnicity, socio-economic status, premorbid disability, family history;

Stroke-related risk factors - smoking, alcohol, hypertension, diabetes, atrial fibrillation, transient ischaemic attack, ischaemic heart disease, medications prior to stroke;

Initial impairments - urinary incontinence, motor weakness, loss of consciousness [Glasgow Coma scale], visuospatial neglect, dysphasia and dysphagia;

Stroke phenotypes - pathological (ischaemic, intracerebral haemorrhage, subarachnoid haemorrhage), clinical (Oxfordshire Community Stroke Project classification) [33], aetiological (modified TOAST classification) [34];

Resources - bed type, length of stay, use of investigations, risk factor management. 
The Register currently has over 2700 incident cases and is probably the largest European stroke register to date with good phenotype data. All patients are followed up annually.

\section{Denominator population and socioeconomic deprivation data}

Revised 2001 census population counts by sex and fiveyear age band will be obtained from ONS at the output area level (mean count 297, SD 72) and used for small area level analyses. We will consult local borough councils regarding the accuracy of population counts and residential developments and compare counts with primary care trust population registers derived from the Exeter system before using the data. The 2001 census based Townsend deprivation index and Index of Multiple Deprivation will be used as indices of deprivation at the small area (census output area) level.

\section{GIS data linkage and modelling}

A Geographical Information System (GIS) will be used to link air pollution and meteorological data with health event data [35,36]. GIS is excellent for linkage of data from different spatial frameworks and analytical work will be carried out to examine a number of options for linkage. For linkage of monitored air pollution data, one approach will be to create buffers with varying radii around each monitoring site and assign pollution values to all cases (residential postcodes) located within these buffers. Where buffers overlap, postcodes may be linked to the nearest monitor. Another approach is to either calculate the daily average of all monitoring stations within the South London study area or spatial interpolation within the area to create a pollution surface, from which pollution levels may be assigned to postcodes. A further issue is the selection of monitors for linkage. We will examine the spatial distribution and population coverage of the different types of monitors and consult with DEFRA before deciding on the monitored pollution information to be used. We will use areal interpolation methodology we have refined for interpolating modelled pollution data from grid squares to output areas for small area level analyses $[35,36]$. For cohort study members, we plan to use buffers of varying radii around residential postcodes to examine the effect of spatially averaged exposure levels, in addition to assigning exposure based on point in polygon methodology. Monitoring stations for meteorological data are generally not co-located with air pollution monitoring stations. We will examine the distribution of these stations and investigate options including buffer-based and spatial interpolation methods as described above before choosing an appropriate method for linking meteorological data to stroke event residential postcodes.

\section{Statistical modelling and analysis}

The case-crossover dataset will be analysed using conditional logistic regression within SAS. Pollutants and meteorological variables will be examined as continuous and as categorical variables. We will examine a range of sampling strategies for obtaining control values and a range of lag days and cumulative lag periods. Effect modification due to sex, age, temperature and socioeconomic deprivation will then be investigated. Differential effects of pollutants will be examined using multi-pollutant models and an alternative method, control by matching, developed by Schwartz [37]. Conditional autoregressive spatial modelling within a Bayesian hierarchical framework will be used for spatial analysis, with expected counts standardised for age and sex, and deprivation included as a covariate. Models will be checked for sensitivity to priors. Cox's proportional hazards modelling will be used for survival analysis, adjusting for a range of prognostic factors. We will then model uncertainty in exposure assessment using a conditional logistic approach within a Bayesian framework based on Bayesian approaches to errors in variables. There are at least four sources of uncertainty in exposure measurement in the acute effects analyses - distance of residential postcode from monitoring site, measurement precision, variation in levels within a day for daily average measures, and variation within a cumulative exposure period.

\section{Other issues}

Ethics approval for research using the South London Stroke Register has been granted by the St. Thomas' Hospital Research Ethics Committee. There will be no patient contact and no intervention involved in this research project.

Air pollution is potentially a modifiable risk factor for stroke. The study will provide robust quantified evidence regarding the effects of outdoor air pollution on stroke incidence, phenotypes and survival. This will provide policy-makers at national and international levels with detailed information and evidence to be used in conjunction with other available evidence when decisions are made regarding national air quality standards and stroke prevention.

The results will be presented at local, national and international meetings and submitted for publication in peerreviewed journals. Details of the work and results will also be placed on the Public Health GIS Unit's website [38] which regularly attracts interest from an international audience. 


\section{Funding}

The project is funded by the Colt Foundation [39]. It commenced in October 2005 and is funded for a two year period.

\section{References}

I. Wolfe CDA: The impact of stroke. Brit Med Bull 2000, 56:275-286.

2. Maheswaran R, Haining RP, Brindley P, Law J, Pearson T, Fryers PR, Wise S, Campbell MJ: Outdoor air pollution and stroke in Sheffield, United Kingdom - a small-area level geographical study. Stroke 2005, 36:239-243.

3. Peters A, Dockery DW, Muller JE, Mittleman MA: Increased particulate air pollution and the triggering of myocardial infarction. Circulation 200I, 103:2810-28I5.

4. Peters A, von Klot S, Heier M, Trentinaglia I, Hormann A, Wichmann $\mathrm{HE}$, Lowel H: Cooperative HealthResearch in the Region of Augsburg Study Group: Exposure to traffic and the onset of myocardial infarction. N Engl I Med 2004, 35 I: I72I-I730.

5. Logan WPD: Mortality in the London fog incident, 1952. Lancet 1953, I:336-338.

6. Glasser M, Greenburg L, Field F: Mortality and morbidity during a period of high levels of air pollution. Arch Environ Health 1967, I 5:684-694.

7. Abercrombie GF: December fog in London and the emergency bed service. Lancet 1953, I:234-235.

8. Moolgavkar $\mathrm{SH}$ : Air pollution and daily mortality in three US counties. Environ Health Perspect 2000, 108:777-784.

9. Hoek G, Brunekreef B, Fischer P, van Wijnen J: The association between air pollution and heart failure, arrhythmia, embolism, thrombosis, and other cardiovascular causes of death in a time series study. Epidemiology 200 I, I 2:355-357.

10. Hong YC, Lee JT, Kim H, Ha EH, Schwartz J, Christiani DC: Effects of air pollutants on acute stroke mortality. Environ Health Persp 2002, I 10:187-191.

II. Kan H, Jia J, Chen B: Acute stroke mortality and air pollution: new evidence from Shanghai, China. J Occup Health 2003, 45:32I-323.

12. Hong YC, Lee JT, Kim H, Kwon HJ: Air pollution: a new risk factor in ischemic stroke mortality. Stroke 2002, 33:2165-2169.

13. Kim H, Kim Y, Hong YC: The lag-effect pattern in the relationship of particulate air pollution to daily mortality in Seoul, Korea. Int J Biometeorol 2003, 48:25-30.

14. Wordley J, Walters S, Ayres JG: Short term variations in hospital admissions and mortality and particulate air pollution. Occup Environ Med 1997, 54:108-116.

15. Wong TW, Tam WS, Yu TS, Wong AH: Associations between daily mortalities from respiratory and cardiovascular diseases and air pollution in Hong Kong, China. Occup Environ Med 2002, 59:30-35.

16. Ponka A, Virtanen M: Low-level air pollution and hospital admissions for cardiac and cerebrovascular diseases in Helsinki. Am J Public Health 1996, 86: I273-I 280.

17. Poloniecki JD, Atkinson RW, de Leon AP, Anderson HR: Daily time series for cardiovascular hospital admissions and previous day's air pollution in London, UK. Occup Environ Med 1997, 54:535-540.

18. Moolgavkar $\mathrm{SH}$ : Air pollution and hospital admissions for diseases of the circulatory system in three US metropolitan areas. J Air Waste Manage Assoc 2000, 50: I I99-I 206.

19. Ballester F, Tenias JM, Perez-Hoyos S: Air pollution and emergency hospital admissions for cardiovascular diseases in Valencia, Spain. J Epidemiol Community Health 200I, 55:57-65.

20. Tsai SS, Goggins WB, Chiu HF, Yang CY: Evidence for an association between air pollution and daily stroke admissions in Kaohsiung, Taiwan. Stroke 2003, 34:26 I 2-26I6.

21. Barer D, Ebrahim S, Smith C: Factors affecting day to day incidence of stroke in Nottingham. BMJ 1984, 289:662.

22. Le Tertre A, Medina S, Samoli E, Forsberg B, Michelozzi P, Boumghar A, Vonk JM, Bellini A, Atkinson R, Ayres JG, Sunyer J, Schwartz J, Katsouyanni K: Short-term effects of particulate air pollution on cardiovascular diseases in eight European cities. J Epidemiol Community Health 2002, 56:773-779.
23. Maheswaran R, Elliott P, Strachan DP: Socioeconomic deprivation, ethnicity and stroke mortality in Greater London and South-East England. J Epidemiol Community Health I997, 5 I:|27-|3|.

24. Seaton A, MacNee W, Donaldson K, Godden D: Particulate air pollution and acute health effects. Lancet I995, 345: I 76-I 78.

25. Maheswaran R, Elliott P: Stroke mortality associated with living near main roads in England and Wales - a geographical study. Stroke 2003, 34:2776-2780.

26. Schwartz J: What are people dying of on high air pollution days? Environ Research 1994, 64:26-35.

27. Strachan DP: Ventilatory function as a predictor of stroke. $B M$ I991, 302:84-87.

28. Maclure $M$ : The case-crossover design: a method for studying transient effects on the risk of acute events. Am J Epidemiol 1991, 133:144-153.

29. Bateson T, Schwartz J: Selection bias and confounding in casecrossover analyses of environmental time-series data. Epidemiology 200 I, I 2:654-66 I

30. Haining RP: Spatial data analysis: theory and practice Cambridge: Cambridge University Press; 2003.

31. Spiegelhalter D, Thomas A, Best N, Lunn D: WinBUGS User Manual Version I.4 Cambridge: Medical Research Council Biostatistics Unit; 2003.

32. Stewart J, Dundas R, Howard R, Rudd A, Wolfe CDA: Ethnic differences in stroke incidence - The South London Stroke Register. BMJ I999, 3 I 8:967-97I.

33. Bamford J, Sandercock P, Dennis M, Burn J, Warlow C: Classification and natural history of clinically identifiable subtypes of cerebral infarction. Lancet 1991, 337:1521-1526.

34. Adams HP Ir, Bendixen BH, Kappelle LJ, Biller J, Love BB, Gordon DL, Marsh EE 3rd: Classification of subtype of acute ischemic stroke. Definitions for use in a multicenter clinical trial. TOAST. Trial of Org 10172 in Acute Stroke Treatment. Stroke 1993, 24:35-4I.

35. Brindley P, Maheswaran R, Pearson T, Wise S, Haining RP: Using modelled outdoor air pollution data for health surveillance. In GIS in Public Health Practice Edited by: Maheswaran R, Craglia M. Boca Raton: CRC Press; 2004:| 25-149.

36. Brindley P, Wise SM, Maheswaran R, Haining RP: The effect of alternative representations of population location on the areal interpolation of air pollution exposure. Comput Environ Urban 2005, 29:455-459.

37. Schwartz J: Is the association of airborne particles with daily deaths confounded by gaseous air pollutants? An approach to control by matching. Environ Health Perspect 2004, I | 2:557-56I.

38. Public Health GIS Unit [http://gis.sheffield.ac.uk]

39. The Colt Foundation [http://www.coltfoundation.org.uk]
Publish with Bio Med Central and every scientist can read your work free of charge

"BioMed Central will be the most significant development for disseminating the results of biomedical research in our lifetime. "

Sir Paul Nurse, Cancer Research UK

Your research papers will be:

- available free of charge to the entire biomedical community

- peer reviewed and published immediately upon acceptance

- cited in PubMed and archived on PubMed Central

- yours - you keep the copyright 